\title{
Normal newborn with prenatal suspicion of $X$ chromosome monosomy due to confined placental mosaicism
}

\author{
Danielius Serapinas Prof. M.D. Ph.D. ${ }^{a}$, Daiva Bartkeviciene Assoc. Prof. M.D. Ph.D. ${ }^{b}$, \\ Emilija Valantinaviciene M.D. ${ }^{a}$ and Egle Machtejeviene Assoc. Prof. M.D. Ph.D. ${ }^{c}$
}

\begin{abstract}
The recent introduction of cell-free DNA (cfDNA)-based noninvasive prenatal testing (NIPT) offers pregnant women a more accurate method than traditional serum screening methods for detecting fetal aneuploidies. Clinical trials have demonstrated the efficacy of NIPT for Down, Edwards and Patau syndromes. However NIPT approaches that take advantage of single-nucelotide polymorphism (SNP) information potentially allow theidentification of triploidy, chromosomal microdeletion syndromes and other unusual genetic variants. To highlight this approach of NIPT we present a rare case of confined placental $X$ chromosome monosomy mosaicism that was prenatally suspected with a single-nucleotide polymorphismbased noninvasive prenatal test. The results of invasive tests (amniocentesis) showed small proportion of $\mathrm{X}$ chromosome mosaicism (45, X[5]/46, XX[95]). After birth karyotype of the girl revealed no abnormalities (46XX), confirming that mosaicism was limited to the placenta. These results highlight the need of patient's informed consent and thorough pretest and postest counseling to ensure that they understand the limitations and advantages of the tests and the implications of the results. Key words: X chromosome disorder, counseling, karyotyping, mosaicism, placenta.
\end{abstract}

http:/ /dx.doi.org/10.5546/aap.2016.eng.e362

\section{INTRODUCTION}

Non-invasive prenatal testing (NIPT) for aneuploidy using cell-free DNA in maternal plasma is revolutionizing prenatal screening

a. Department of Genetics and molecular medicine, Medical Academy, Lithuanian University of Health Sciences, Kaunas, Lithuania.

b. Department of Obstetrics and gynecology, Vilnius University, Vilnius, Lithuania.

c. Department of Obstetrics and gynecology, Medical Academy, Lithuanian University of Health Sciences, Kaunas, Lithuania.

E-mail Address:

Dr. Danielius Serapinas: dserapinas@gmail.com

Funding: None.

Conflict of interest: None.

Received: 2-18-2016

Accepted: 4-11-2016 and diagnosis; it was introduced in clinical practice in 2011. This approach had the purpose to minimize the need of invasive prenatal testing like villus sampling or amniocentesis, that were used as conventional prenatal diagnostic procedure when biochemical or ultrasound markers screening suggested increased risk of aneuploidy. A more recent NIPT method using a single-nucleotide polymorphism (SNP)-based method along with sophisticated informatics, can resolve this potential source of false positive results that is an especially problematic aspect in prenatal biochemical screening. This NIPT approach identifies the presence of additional fetal haplotypes, indicative of triploidy or even mosaicism. ${ }^{1}$

In the current context it is important to distinguish prenatal diagnosis of aneuploidy from antenatal screening. A diagnostic test performed as chorionic villi, amniotic fluid or fetal blood needs to have very few false negatives (aneuploid pregnancies misdiagnosed as euploid) and false positives (euploid pregnancies misdiagnosed as aneuploid), since the result will inform the decision as to whether to terminate the pregnancy. ${ }^{2}$ In contrast, antenatal screening does not aim to be definitive; rather, it is designed to identify women who are at sufficiently high risk of common aneuploidies as to warrant invasive prenatal diagnosis. Main syndromes, that can be identified with NIPT are Down (21 trisomy), Edwards (18 trisomy), Patau (13 trisomy), and Turner ( $\mathrm{X}$ monosomy) syndrome. ${ }^{3}$ NIPT test has a great sensitivity (true positive rate) and specificity (true negative rate) rates for these genetic disorders. Specificity is higher than 99 percent for the most common trisomy. The highest rate of sensitivity is for Down (99 percent) and Edward (97-99 percent) syndromes and lower for Patau (87-99 percent) and Turner (92-95 percent) syndromes. ${ }^{4}$ We present a case of extraordinary prenatal genetic test results (45, X[5]/46, XX[95]). The outcome of the pregnancy was a healthy girl with a normal karyotype that confirmed the diagnosis of confined placental mosaicism (CPM). 


\section{CASE REPORT}

A 36-year-old primigravid woman was referred for NIPT at 17 weeks of gestation because the first trimester biochemical screening test results showed high risk for 21 trisomy (1:50). Biochemical testing revealed a high level of free $\beta$-hCG and a reduced PAPP-A (1.72 MoM and $0.63 \mathrm{MoM}$, respectively). Prenatal ultrasound at 12 weeks of gestation showed normal development of the fetus (NT $1.6 \mathrm{~mm}$ ). Maternal blood samples $(10 \mathrm{~mL})$ for NIPT were drawn to the blood collection tubes and processed at Natera (San Carlos, CA) within 6 days of collection. Testing was performed as previously described using validated laboratory methodologies (cell free DNA -cfDNA- isolation, polymerase chain reaction amplification targeting 19,488 SNPs, high-throughput sequencing, and analysis using the Next-generation Aneuploidy Test Using SNPs [NATUS] algorithm). ${ }^{1,2}$

Patient's NIPT Panorama test was performed. Fetal fraction was $6 \%$ and test results were negative for 21, 18, 13 trisomies and triploidy. The summary of the test report stated that atypical findings could be compatible with fetal mosaicism for $\mathrm{X}$ chromosome. However, this finding is not conclusive and other possibilities could not be ruled out. Follow-up testing and counseling was recommended. The NIPT test detects single nucleotide polymorphisms (SNPs) in maternal and fetal DNA. The maternal contribution is used only as an input to the fetal-risk calculation. Therefore, incidental findings of maternal cancer risk or abnormal numbers of sex chromosomes are not an issue. ${ }^{3}$ This is the reason why the presence of an additional fetal haplotype was identified as a possible effect of $X$ chromosome mosaicism.

The frequency and significance of placental mosaicism suggest that this condition could result in either false-positive or false-negative NIPT results. NIPT could fail to identify some viable trisomy cases that have a substantial population of abnormal fetal, but not placental, cells. This could lead to an apparently false negative test result. Conversely, the mostly abnormal trophoblasts with normal fetal cells could lead to an apparently false-positive result. Moreover, if chromosomally abnormal trophoblasts are more likely to undergo apoptosis, NIPT could potentially identify cases in which the proportion of abnormal placental cells is low. ${ }^{2}$ This issue is of high concern, keeping in mind that approximately $14 \%$ of all cytogenetic abnormalities identified through karyotyping of amniotic fluid cells are mosaic. $^{2}$

It is important to remember that the sequencing is performed on total DNA, which is a combination of maternal and fetal DNA. At this time, the algorithm couldn't distinguish if it was maternal or fetal mosaicism. It was possible

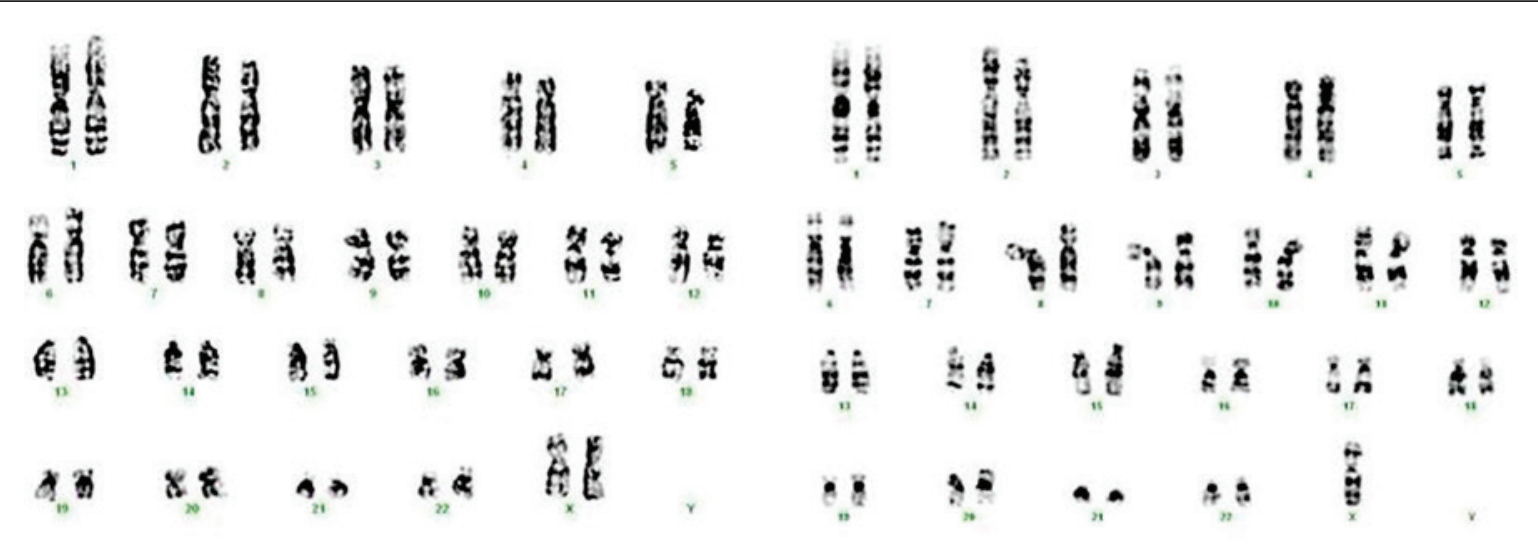

A

B

Confined placental mosaicism of X chromosome monosomy with 95\% 46, XX (A) and 5\% of 45, X (B) cells confirmed by amniocyte karyotyping. 
that the pregnant woman herself was mosaic for $X$ chromosome. Because of that we performed her karyotyping and it came back normal $(46, X X)$. Afterwards, genetic amniocentesis was performed and the results showed that amniocytes had a small proportion of $X$ chromosome mosaicism. Karyotype of the fetus was 45, X [5]/46, $X X$ [95] (Figure 1). The figure shows that 5\% of the cells only have a single $X$ chromosome, suggesting Turner syndrome ( $\mathrm{X}$ monosomy) and the rest of the remaining cells (95\%) have two $\mathrm{X}$ chromosomes, confirming normal female karyotype.

As a result, the newborn showed no phenotypic abnormalities. A healthy baby girl was delivered at 39 weeks, weight was $3020 \mathrm{~g}$ and length was $52 \mathrm{~cm}$, Apgar 10-10. Cytogenetic analysis was performed from the peripheral blood sample (100 mitotic cells) of the newborn and karyotype was of a normal female $(46, X X)$. This data suggests that $X$ monosomy mosaicism could be a complete placental mosaicism. However, we did not test other tissues of the newborn (e.g. skin fibroblasts), that could possibly have a small amount of cells with an X monosomy.

\section{DISCUSSION}

The novelty of this case is that for the first time NIPT (using single-nucleotide polymorphism) suspected confined placental mosaicism (CPM). It was possible because the NATUS algorithm also considers parental genotypic information, crossover frequency data, and possible fetal chromosome copy numbers (monosomy / disomy/trisomy) at the evaluated polymorphic loci. These results demonstrate the sensitivity of the SNP-based sequencing analysis for detecting specific characteristics of cell-free DNA in a complex mixture. ${ }^{5}$ On the one hand, cytogenetic analysis of amniocytes or villi obtained via invasive procedures is currently the reference standard for aneuploidy classification; a karyotype performed on a limited number of cells also cannot rule out low-level mosaicism. ${ }^{6}$ On the other hand, even invasive testing cannot distinguish between fetal mosaicism and CPM.

According to Taylor ${ }^{7}$ chorionic villus sampling and amniocentesis involves the removal of a sample of extraembryonic mesoderm or extraembryonic ectoderm, which is not part of the fetus proper. Because of that there is no direct study of chromosomes of the fetus during pregnancy, unless a cordocentesis - fetal blood sampling. Furthermore a complication as miscarriage can occur during invasive prenatal diagnostic methods especially in no expert's hands; amniocentesis can cause $1 \%$ of miscarriage. Non-invasive test like NIPT should be considered as more acceptable and safer test for fetal karyotyping.

Despite high accuracy, prenatal diagnostic procedures have also limitations. In our case at first biochemical test risk for 21 trisomy was increased, afterword genetic tests revealed $X$ monosomy mosaicism, but at the end it was delivered a healthy girl. So the obligation of doctors to inform patients is inseparable from the requirement to receive informed consent. The main requirements for the informed consent include rationality, sufficient and clear information, free will and the form of consent conforming to the legal acts. Prenatal testing is an integral part of pregnancy care, which is aimed to verify the proper development of the fetus or to identify the potential hereditary or chromosomal diseases. If data are received and is very difficult to predict the phenotype of the future baby, close monitoring and surveillance during the pregnancy could be reassuring future parents.

In conclusion, all patients should receive thorough pretest counseling to ensure that they understand the limitations of the test and the implications of the results. As the experience accumulates with more extraordinary cases, it is possible that NIPT will replace current screening protocols and become the primary screening and ultimately a noninvasive diagnostic test for fetal aneuploidy.

\section{REFERENCES}

1. Cuckle H, Benn P, Pergament E. Cell-free DNA screening for fetal aneuploidy as a clinical service. Clin Biochem 2015;48(15):932-41.

2. Benn P, Cuckle H, Pergament E. Non-invasive prenatal testing for aneuploidy: current status and future prospects. Ultrasound Obstet Gynecol 2013;42(1):15-33.

3. Gross SJ, KarehtS, Ryan A. Prenatal screening: focus on more specific fetal testing. Nature 2015;523(7560):290.

4. Allyse M, Minear MA, Berson E, Sridhar S, et al. Noninvasive prenatal testing: a review of international implementation and challenges. Int $J$ Womens Health 2015;7:113-26.

5. BennP, Borrell A,ChiuRW,CuckleH, etal.Positionstatement from the Chromosome Abnormality Screening Committee on behalf of the Board of the International Society for Prenatal Diagnosis. Prenat Diagn 2015;35(8):725-34.

6. Bianchi DW, Platt LD, Goldberg JD, Abuhamad AZ, et al. Genome-wide fetal aneuploidy detection by maternal plasma DNA sequencing. Obstet Gynecol 2012;119(5):890-901.

7. Taylor TH, Gitlin SA, Patrick JL, Crain JL, et al. The origin, mechanisms, incidence and clinical consequences of chromosomal mosaicism in humans. Hum Reprod Update 2014;20(4):571-81. 